\title{
In-vivo study of synthetic and natural food colors effect on biochemical and immunity parameters
}

\author{
Sadaf SHAKOOR ${ }^{1,2 *}$ (D), Amin ISMAIL ${ }^{2,3}$, Mohd Redzwan SABRAN ${ }^{2}$, Norhafizah MOHTARRUDIN ${ }^{4}$, \\ Ubedullah KAKA ${ }^{5}$, Muhammad NADEEM ${ }^{6}$
}

\begin{abstract}
Incorporation of the coloring agent, tartrazine and curcumin affects healthy physiological system leading to changes in hematology, biochemistry and enzymatic activity. Consequences of oral administration of tartrazine and curcumin were explored for 15, 30 and 45 days in female adult rat. Two doses were based on the admissible daily intake (ADI) of 9.6 and 96 (high) $\mathrm{mg} / \mathrm{kg} /$ body weight for tartrazine, 3.85 and $38.5 \mathrm{mg} / \mathrm{kg} /$ body weight for curcumin. Phytochemicals such as saponins, glycosides, alkaloids, flavonoids, tannin, carbohydrates and phenolics were present in coloring agents. The renal function tests and lipid profile showed alterations in the values of uric acid, urea, total protein, albumin and cholesterol in all treatment groups. Additionally, the levels of liver enzymes fluctuated after 45 days of treatment with curcumin and tartrazine. Histopathological changes were also recorded in liver and kidney. In contrast, blood biochemistry depicted the significant reduction of RBCs, $\mathrm{Hb}$, $\mathrm{MCH}$, glucose, WBCs, MCHC and LDH at 15, 30 and 45 days. Besides, IgG, IgM and overall antibody GMT at 45 days were significantly increased. Study indicated that ADI and doses up to 10 times of the ADI of food colors intake exerted adverse effects on immune response and altered the biochemical parameters.
\end{abstract}

Keywords: food colors; hematology; antibody; toxicology; biochemistry; immunity.

Practical Application: Food colors may not be used in high dosage for long term.

\section{Introduction}

Food, as a basic need for all human and animals, must become wholesome and safe. It is only nutritive when it is pure, fresh and free from hazardous matters (Jonnalagadda et al., 2004). Food coloring is controversial and premier public hazard affecting the quality of life. Due to illiteracy and poor knowledge of food colors, many industries use banned and synthetic colors to add in varieties of food products (Jeannine, 2003).

On the global scale, the use of colors in food has faced challenges with disagreement, particularly when they are added to food at high doses, which exceeds the recommended dose. The utilization of colors in food confronted with debate when add up into food, specifically in children nutrition, causing allergic reactions, respiratory, liver and kidney disorders (Hashem et al., 2010). In addition, synthetic dye contained various heavy metals such as $\mathrm{Pb}, \mathrm{Hg}$, As, Cu, Ni, Mg, Co, etc (Protima, 2008). Worldwide production of food colors is estimated to be around 8 million tons for each year as well as 2000 different dyes are presently used by food industries (Vijaykumar et al., 2007). The market for natural colorant is growing $10 \%$ per year. Indeed, China, India, UK, and South Korea have the largest supplier market for food colors (Export-Import Bank of India, 2010).
Synthetic food colors are prepared from hydrocarbons mixture and have been certified by the Food and Drug Administration (FDA) to retain food texture and appearance. Artificial food colorants can attain shades as compared to natural (Vojdani \& Vojdani, 2015). Whereas natural coloring produced from plants are costly, non-stable and easily fades, in addition, many researchers examined the adverse effect on metabolism and toxicity induced by the consumption of specific food colors to laboratory animals (Tanaka, 2005; Amin et al., 2010). The general population may be exposed to food colors via ingestion of food and medications, and dermal contact with these compounds via consumer products containing particular or combination of food colors (National Institute for Occupational Safety and Health, 2011). Nevertheless, there is dearth of data about the direct adverse effect of food coloring towards human health.

Many food colors are made from petroleum and coal tar called azo dyes. One of significant azo dye is tartrazine recognized as E102 or FD \& C yellow 5, widely used synthetic food color. Tartrazine is a nitrous derivative (azo class) metabolised to aromatic amines principally by gut microorganisms, azoreductases and liver enzymes playing a minor role in dye reduction (Chung et al., 1992). Tartrazine causes changes in hepatic and renal 
parameters as well as it poses more risk due to the induction of oxidative pressure through the production of free radical and allergic reactions in sensitive individuals at high doses. In fact, the mutagenic effect of DNA by tartrazine has been established through animal studies (Sasaki et al., 2002). Of great concern, food colors were used above the acceptable daily intake (ADI) during various occasions in many developing countries which would cause severe health complications in human (Rao et al., 2006).

Curcumin is one of the natural colorants extracted from the Curcuma longa. It is a yellow-orange pigment and generally known as $\mathrm{E} 100$. The turmeric is a common constituent in gravy spices and has been utilized in asian medication designed for treatment of wide variety of syndromes such as obesity, hypertension, cancer and skin related diseases from many years (Ravindran et al., 2009). It is no longer surprising, therefore, that curcumin is now marketed as a dietary supplement and that numerous experimental trials are ongoing or recruiting volunteers to gauge curcumin activity. However, there is accumulating indication that curcumin may not be so useful and safe. Curcumin does not give short-term toxicity at doses up to $8 \mathrm{~g} /$ day resulted from the human trial. However, curcumin dosage ranges 0.9 to $3.6 \mathrm{~g} / \mathrm{day}$ for 1-4 months produced several negative results containing nausea, diarrhea, increment in serum alkaline phosphatase and lactate dehydrogenase enzymes in humans (Sharma et al., 2004).

Due to industrialization and shortage of time, most of the people utilize packed or ready to cook food items to save their time. In the recent scenario, food colors are difficult to omit from daily food stuff i.e confectionaries, bakery products, canned products, etc. Previous studies researched the metabolic, physiological and toxicological disorders brought by the uncontrolled use of different shade of synthetic and natural colors to male young and small rats and other mammals. In this context, current research has tried to study nutritional profile of tartrazine (Artificial) and curcumin (Natural) and feed containing tartrazine and curcumin and checked their low as well as high dose effect on some physical, biochemical, hematological and immunity parameters in the adult female albino rat at different time duration. According to U.S. Food and Drug Administration (1985) infant animals are more vulnerable to contaminant or carcinogens as compared to older animals. Literature lack the data regarding effect of different food colors on female adult rats and no study related to the qualitative and quantitative phytochemical profile of any food colors is available up to date. We attempted to fill this gap, compare and demonstrate the effects of same yellow shade of curcumin natural colorant artificially replaced by tartrazine colorants on the physiological functions i.e. blood parameters, liver and kidney function tests and immunity level of adult female rats.

\section{Methodology}

\subsection{Food colors utilized in the experiment}

Tartrazine: (CAS 1934-21-0, Purity 86.7\%) was purchased in the form of powder from Sigma Aldrich (USA). Its chemical formula: Trisodium salt: Trisodium 5-hydroxy-1-(4-sulfonatophenyl)-4(E)-(4 sulfonatophenyl) diazenyl-1H-pyrazole-3-carboxylate. Molecular formula: $\mathrm{C}_{16} \mathrm{H}_{9} \mathrm{~N}_{4} \mathrm{Na}_{3} \mathrm{O}_{9} \mathrm{~S}_{2}$, Formula weight: $534.3 \mathrm{~g} / \mathrm{mol}$.
Curcumin: (CAS 458-37-7, Purity: = 95\%) was purchased in the form of powder from Carbosynth, UK. Its chemical formula: 1 , 7-bis (4-hydroxy-3methoxyphenyl)-1E, 6E-heptadiene-3, 5-dione. Molecular formula: $\mathrm{C}_{21} \mathrm{H}_{20} \mathrm{O}_{6}$, Formula weight: $368.4 \mathrm{~g} / \mathrm{mol}$.

\subsection{Animals}

Ninety non pregnant female Sprague Dawley rats of 200.65 \pm 15.20 grams, 6-7 months old (sourced from animal house of University of Agriculture Faisalabad, Pakistan) were divided into 15 groups $(n=6)$ (Figure 1). Rats were kept in individual stainless steel cages at $27 \pm 5{ }^{\circ} \mathrm{C}$ and under good ventilation.

\subsection{Treatment}

Tartrazine and curcumin were used at ADI recommended by JECFA and at 10 times the ADI concentration ( 9.6 and $96 \mathrm{mg} / \mathrm{kg}$-b.w/day for tartrazine and 3.85 and $38.5 \mathrm{mg} / \mathrm{kg}-\mathrm{b} . \mathrm{w} /$ day for curcumin). Dose was converted according to animal equivalent dose using the formula by Nair \& Jacob (2016). The colors at stated doses were incorporated into the standard feed (Faisalabad, Pakistan) with addition of low and high doses of food colors and fed to the animals daily for 15, 30 and 45 days respectively based on the study protocol (Figure 1).

\subsection{Sampling}

Sampling was done at 15, 30 and 45 days to check acute, sub-acute and post-acute toxicity. At the completion of each experimental period, 4-5 mL blood samples were taken through cardiac puncture of the rats by using $23 \mathrm{G}$ needle under general anesthesia. Blood was collected in BD Vacutainer ${ }^{\circledast}$ heparin tubes. Then rats were immediately euthanized through cervical dislocation. The blood was centrifuged using centrifugation (LYC; Centrifuge 80-2, Jiangsu, China) at $3000 \mathrm{rpm}$ and room temperature to collect plasma, which was transferred to the ependoff ans stored at $-20^{\circ} \mathrm{C}$ untill further analysis (within 4 weeks).

One milliliter blood was taken in dry glass centrifuge tubes, let to coagulate and centrifuged at $3500 \mathrm{rpm}$ for $15 \mathrm{~min}$ at room temperature to collect serum to be used for immunity determination.

\subsection{Body weight, feed and water intake}

Body weight of animal was documented before and later the experiment using a weighing scale. The percentage of gain weight was expressed as [(final weight - beginning weight)/ beginning weight] $\times 100$ (El-Malky et al., 2014).

30-35 g/animal feed and 30-35 mL/animal water was provided to the experimental animal.

\subsection{Proximate analysis of feed containing food colors}

Moisture was estimated by the protocol of American Association of Cereal Chemists (2000) method no. 44-15 and procedure No.08-01 was adopted for calculation of ash contents. Crude fat was measured following the protocol of American Association of Cereal Chemists (2000) method no.30-10. Crude fiber was projected by adopting the technique as outlined in American Association of Cereal Chemists (2000) method no. 


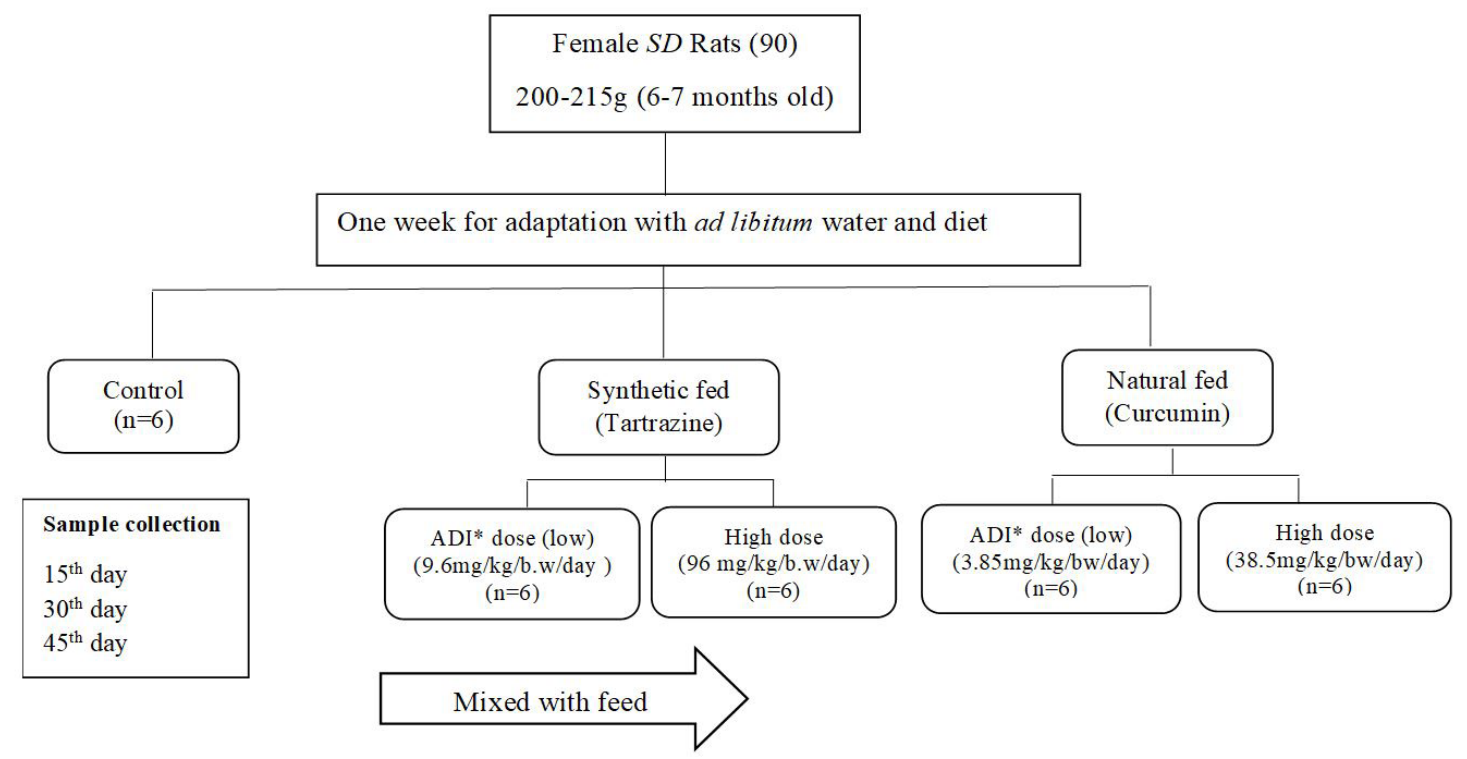

*ADI= Acceptable daily intake

Figure 1. Experimental animals and study protocol.

32-10. The protein content in the feed sample was measured as defined in American Association of Cereal Chemists (2000) method no. 46-10.

\subsection{Preparation of extracts}

Ethanolic, methanolic and aqueous, extracts of feed were prepared by adding 50 grams of tartrazine and curcumin containing feed in $50 \mathrm{~mL}$ of distilled water, ethanol, and methanol respectively, and left overnight. Then the mixture was macerated and stirred for 1 hour using a magnetic stirrer. Later, the mixture was percolated using a Whatman filter paper number 1 and shifted it into a sterile amber color bottles for further analysis.

\subsection{Screening of phytochemicals}

Qualitative analysis of feed containing low and high doses of tartrazine and curcumin

Saponins, anthraquinones and alkaloids were detected by the method of Balabhaskar \& Vijayalakshmi, (2015). Amino acids and total carbohydrates were detected by the method of Tiwari et al. (2011). Xanthoproteic test was used to detect the proteins. Coumarins and steroids were identified by adopting the procedure of Harsha et al. (2013). Flavonoids, phenols and tannins were detected according to the protocol of Ben et al. (2013). Anthocyanins were measured by the procedure of Savithramma et al. (2011). Fixed oil was checked through the method of Ghongade (2013). Glycosides were checked using the procedure of Sathyaprabha et al. (2011). Resin was tested by adopting protocol by Shabi et al. (2014). Salkowski test was used for the detection of terpenoids Veerachari \& Bopaiah (2011).
Quantitative screening of feed containing low and high doses of tartrazine and curcumin

Total phenolic and flavonoids contents were determined spectrophotometrically adopting to the method of Amir et al. (2011). Total carbohydrate content was determined by procedure of Kumar et al. (2012). Determination of total alkaloids was done following the protocol of Shamsa et al. (2008). Total tannins were evaluated by adopting the procedure of Tamilselvi et al. (2012).

\subsection{Blood biochemistry}

\section{Hematology}

By the end of the experimental periods, one to two $\mathrm{mL}$ blood samples were collected through cardiac puncture of the rats by using $23 \mathrm{G}$ needle under general anesthesia Blood was collected in $\mathrm{BD}$ Vacutainer ${ }^{\circ}$ heparin tubes and immediately analyzed using an automatic hematology analyzer (Sysmex K-1000, Minnesota, USA) in order to determine red blood cell count (RBC), white blood cells (WBC), hemoglobin concentration (HGB), hematocrit $(\mathrm{HT})$, mean corpuscular hemoglobin $(\mathrm{MCH})$, mean corpuscular volume (MCV), mean corpuscular hemoglobin concentration (MCHC), platelets (PLT), neutrophils, lymphocytes, monocytes, and eosinophils (Zhang et al., 2018).

\section{Liver function enzymes}

ELISA sets (DiaSys; Diagnostic system GmbH, Holzheim, Germany) were used to measure the alanine transaminase (ALT), aspartate transaminase (AST), alkaline phosphatase (ALP) and lactate dehydrogenase (LDH) according to the manufacturer guidelines. 


\section{Renal function tests}

BUN (Blood urea nitrogen) creatinine, uric acid, albumin, bilirubin, globulin, AG ratio, total proteins were measured in blood samples using commercially available diagnostic sets (DiaSys; Diagnostic system GmbH, Holzheim, Germany) by adopting the manufacturer guidelines.

\section{Blood glucose and cholesterol}

Glucose, cholesterol and triglycerides were measured in blood samples using commercially available diagnostic sets (DiaSys; Diagnostic system $\mathrm{GmbH}$, Holzheim, Germany) by adopting the manufacturer guidelines.

\subsection{Immunity testing by antibody response to sheep $R B C s$}

Antibody response to sheep RBCs was determined based on the method adopted by Khatoon et al. (2013). For the purpose of SRBC's Sheep blood had withdrawn using a syringe comprising EDTA which was wash away thrice through normal saline at $1500 \mathrm{rpm}$ (SRBC; obtained from sheep kept by the Department of Microbiology in University of Agriculture (Faisalabad, Pakistan). Afterwards, 3\% suspension of eroded SRBCs was prepared in normal saline. The suspension of SRBCs was intravenously inoculated to 6 rats per replicate at 36th and 38th day (primary and secondary shot) of experimental period in order to get antibodies titer of 45 day phase. Blood was collected at 45 th day of experiment. Serum separated from blood and kept at $-20{ }^{\circ} \mathrm{C}$ till it was investigated intended for antibody response. All data were expressed in terms of $\left(\log _{2}\right)$.

\subsection{Statistical analysis}

The statistical analysis was done by using SPSS software version 25. One-way analysis of variance was applied followed by Tukey Kramer multiple comparison post- hoc test as analyzed by Lin et al. (2019).

\section{Results}

\subsection{Proximate profiling}

Proximate composition was performed to determine the quality of food colors, change in nutrients of food colors and feed before and after incorporation of a low and high dose of food colors. $100 \mathrm{~g}$ of tartrazine contained $236 \mathrm{kcal}, 39.31 \mathrm{~g}$ ash, 1.86 moisture, 31.17 carbohydrates. $100 \mathrm{~g}$ of curcumin contained $453 \mathrm{kcal}$ energy, $0.1 \mathrm{~g}$ ash, $0.22 \mathrm{~g}$ moisture, $0.26 \mathrm{~g}$ protein, and carbohydrate $99.42 \mathrm{~g}$. Proximate value of feed containing studied food color is shown in Table 1.

\subsection{Phytochemicals profiling}

Table 2 shows the presence of terpenoids and saponins in all extracts of control and treated feed samples. However, glycosides were positive in ethanolic and methanolic extracts of all feed sample compared to aqueous. Alkaloids were found in aqueous extract of treated feed sample compared to control feed, ethanolic and methanolic extracts. Flavonoids were positive in aqueous extract of all feed samples whereas flavonoids were also positive in ethanolic and methanolic extract of control feed compared to treated feed samples (LT, HT, LC and HC). Moreover, tannin was positive in aqueous extract of all feed sample and in methanolic extracts of control, LT, HT compared to LC, HC and ethanolic extract. On the one hand, anthraquinones were positive in ethanolic and methanolic extract of $\mathrm{HC}$ feed sample compared to aqueous extract and C, LT, HT and LC feed samples. Tannin was less in a water extract of all feeds compared to control but higher in ethanolic and methanolic extract feeds compared to control feed (Table 3).

Aqueous extract of HT, LC contained fewer phenolics content, one the other hand, LT, and HC showed higher phenolics compared to control. Phenolics were low in the LC group compared with control whereas LT, HT and HC showed higher content of phenolics. However, methanolic extract of all feeds showed high phenolics compared to control. Flavonoids were high in the water extract of LC and HC, ethanolic extract of LT, HT and HC, methanolic extract of LT, HT, LC and HC compared to their control feed samples (Table 3).

\subsection{Feed intake, water intake and body weight parameters}

Body weight gain \%age of all treated groups (low tartrazine, high tartrazine, low curcumin, and high curcumin) significantly increased at 30 days and 45 days compared to control (Table 4). The water intake was significantly increased in LT30, HT30 and LT45. However, there was no significant difference between treatment groups (LT vs Ht and LC vs HC). The feed intake was significantly decreased after 15 days, 30 day and 45 days (Table 4).

\subsection{Hematological parameters}

There was non-significant lower level of RBCs, $\mathrm{Hb}$ and haematocrit, but WBCs were significantly higher in LT30 and HT30 treated animals. Consumption of curcumin and tartrazine exposed significant $(\mathrm{p} \leq 0.05)$ low level in MCV of the treated group after 30 and 45 days while MCV was found high in all groups of 15days compared to control.

$\mathrm{MCH}$ was significantly lower in LT15, LT30, HT30 and HT45 group. Considerable ( $\mathrm{p} \leq 0.05)$ increment was observed in $\mathrm{MCHC}$ in treatment group of 30 and 45 days. Whereas MCHC was decreased in LT15. HT15 and HC 15 compared to control.

Consumption of tartrazine significantly increased the neutrophills in LT30 and HT30, non-significantly increased in LT45 and HT45. Lymphocytes was found significantly

Table 1. Proximate composition of food colors containing feed.

\begin{tabular}{lccccl}
\hline & $\begin{array}{c}\text { Moisture } \\
\%\end{array}$ & Ash \% & Fiber \% & $\begin{array}{c}\text { Protein } \\
\%\end{array}$ & Fat \% \\
\hline Control & 25.8 & 5.75 & 2.75 & 19.68 & 4 \\
Low Tartrazine & 27.1 & 5.3 & 1.6 & 17.5 & 4.18 \\
High Tartrazine & 10.23 & 7.26 & 3.05 & 21.87 & 4.05 \\
Low Curcumin & 8.43 & 5.94 & 3.4 & 21.85 & 3.5 \\
High Curcumin & 8.45 & 5.3 & 3.85 & 20.23 & 5.1 \\
\hline
\end{tabular}


Original Article

Shakoor et al.

Table 2. Qualitative analysis of feed containing low and high doses of tartrazine and curcumin.

\begin{tabular}{|c|c|c|c|c|c|c|c|c|c|c|c|c|c|c|c|}
\hline \multirow{2}{*}{ Compounds } & \multicolumn{5}{|c|}{ Water Extract } & \multicolumn{5}{|c|}{ Ethanolic Extract } & \multicolumn{5}{|c|}{ Methanolic Extract } \\
\hline & $\mathrm{C}$ & LT & HT & LC & $\mathrm{HC}$ & $\mathrm{C}$ & LT & HT & LC & $\mathrm{HC}$ & $\mathrm{C}$ & LT & HT & LC & $\mathrm{HC}$ \\
\hline Alkaloids & -ve & +ve & $+v e$ & $+v e$ & + ve & -ve & -ve & -ve & -ve & -ve & -ve & -ve & -ve & -ve & -ve \\
\hline Amino acids & -ve & -ve & -ve & -ve & -ve & -ve & -ve & -ve & -ve & -ve & -ve & -ve & -ve & -ve & -ve \\
\hline Anthocyanins & -ve & -ve & -ve & -ve & -ve & -ve & -ve & -ve & -ve & -ve & -ve & -ve & -ve & -ve & -ve \\
\hline Anthraquinones & -ve & -ve & -ve & -ve & -ve & -ve & -ve & -ve & -ve & + ve & -ve & -ve & -ve & -ve & +ve \\
\hline Carbohydrates & -ve & -ve & -ve & -ve & -ve & -ve & -ve & -ve & -ve & -ve & -ve & -ve & -ve & -ve & -ve \\
\hline Cumarins & $+\mathrm{ve}$ & $+\mathrm{ve}$ & + ve & + ve & $+\mathrm{ve}$ & + ve & $+\mathrm{ve}$ & + ve & + ve & $+\mathrm{ve}$ & + ve & $+v e$ & + ve & $+\mathrm{ve}$ & +ve \\
\hline Fixed oils & -ve & -ve & -ve & -ve & -ve & -ve & -ve & -ve & -ve & -ve & -ve & -ve & -ve & -ve & -ve \\
\hline Flavonoids & $+\mathrm{ve}$ & $+\mathrm{ve}$ & $+\mathrm{ve}$ & $+\mathrm{ve}$ & $+\mathrm{ve}$ & $+\mathrm{ve}$ & -ve & -ve & -ve & -ve & $+\mathrm{ve}$ & -ve & -ve & -ve & -ve \\
\hline Glycosides & -ve & -ve & -ve & -ve & -ve & + ve & $+\mathrm{ve}$ & + ve & $+\mathrm{ve}$ & $+\mathrm{ve}$ & $+v e$ & $+v e$ & $+v e$ & + ve & $+\mathrm{ve}$ \\
\hline Phenols & -ve & -ve & -ve & -ve & -ve & -ve & -ve & -ve & -ve & -ve & -ve & -ve & -ve & -ve & -ve \\
\hline Proteins & -ve & -ve & -ve & -ve & -ve & -ve & -ve & -ve & -ve & -ve & -ve & -ve & -ve & -ve & -ve \\
\hline Resins & -ve & -ve & -ve & -ve & -ve & -ve & -ve & -ve & -ve & -ve & -ve & -ve & -ve & -ve & -ve \\
\hline Saponins & $+v e$ & $+v e$ & $+\mathbf{v e}$ & $+v e$ & $+v e$ & $+v e$ & $+v e$ & $+\mathrm{ve}$ & $+v e$ & + ve & $+v e$ & $+v e$ & $+v e$ & $+v e$ & +ve \\
\hline Steroids & -ve & -ve & -ve & -ve & -ve & -ve & -ve & -ve & -ve & -ve & -ve & -ve & -ve & -ve & -ve \\
\hline Tannins & $+v e$ & $+v e$ & $+\mathbf{v e}$ & $+v e$ & $+v e$ & -ve & -ve & -ve & -ve & -ve & $+v e$ & $+v e$ & $+v e$ & -ve & -ve \\
\hline Terpenoids & -ve & -ve & -ve & -ve & -ve & -ve & -ve & -ve & -ve & -ve & -ve & -ve & -ve & -ve & -ve \\
\hline
\end{tabular}

$\mathrm{C}=$ Control; LT = Low Tartrazine; HT= High Tartrazine; LC= Low Curcumin; HC= High Curcumin.

Table 3. Quantitative analysis of feed containing low and high doses of tartrazine and curcumin.

\begin{tabular}{|c|c|c|c|c|c|c|c|c|c|c|c|c|c|c|c|}
\hline \multirow{2}{*}{ Compounds } & \multicolumn{5}{|c|}{ Water Extract } & \multicolumn{5}{|c|}{ Ethanolic Extract } & \multicolumn{5}{|c|}{ Methanolic Extract } \\
\hline & C & LT & HT & LC & $\mathrm{HC}$ & $\mathrm{C}$ & LT & HT & LC & $\mathrm{HC}$ & $\mathrm{C}$ & LT & HT & LC & $\mathrm{HC}$ \\
\hline Carbohydrate mg/dL & 2686 & 3669 & 3354 & 3404 & 3467 & 2867 & 3691 & 3691 & 3336 & 3550 & 2965 & 3557 & 2909 & 3503 & 3691 \\
\hline Tannins $\mathbf{g} / \mathbf{m L}$ & 293.5 & 291 & 262.5 & 267 & 270 & 273.5 & 288 & 279 & 279.5 & 291 & 282 & 288.5 & 287.5 & 295.5 & 293 \\
\hline Phenolics $\mu \mathrm{g} / \mathrm{mL}$ & 60.6 & 82.5 & 57.6 & 53.6 & 61.0 & 109.7 & 109.5 & 111.9 & 99.3 & 112.9 & 102.4 & 108.5 & 108.6 & 107.3 & 114.2 \\
\hline Alkaloids mg/mL & 297 & 84.6 & 52.6 & 20.6 & 82.3 & 518 & 78.33 & 321.33 & 8.67 & 16 & 502.67 & 160 & 126.67 & 228 & 19 \\
\hline Flavonoids $\mu \mathrm{g} / \mathrm{mL}$ & 122.4 & 76.5 & 60.2 & 262 & 169.5 & 241.5 & 256.7 & 283.9 & 210.2 & 403.5 & 182.7 & 225.8 & 268.4 & 219.7 & 403.5 \\
\hline
\end{tabular}

Table 4. Initial, final weight, water and feed intake of treated animals as compared to control.

\begin{tabular}{|c|c|c|c|c|}
\hline Days & Food color Exposure & Body weight gain (\%) & Water Intake (mL) & Feed Intake (g) \\
\hline \multirow[t]{5}{*}{15} & Control & $0.99 \pm 0.49^{\mathrm{e}}$ & $161.20 \pm 4.34^{\mathrm{a}}$ & $118.20 \pm 6.81^{\mathrm{a}}$ \\
\hline & LT & $0.68 \pm 0.30^{\mathrm{e}}$ & $153.26 \pm 9.26$ abcde & $103.20 \pm 2.52^{\mathrm{e}}$ \\
\hline & HT & $0.84 \pm 0.58^{\mathrm{e}}$ & $157.33 \pm 4.23^{\mathrm{ab}}$ & $106.73 \pm 9.25^{\text {bcde }}$ \\
\hline & LC & $1.68 \pm 0.59^{\mathrm{e}}$ & $156.93 \pm 5.63^{\mathrm{ab}}$ & $107.93 \pm 4.47$ bcde \\
\hline & $\mathrm{HC}$ & $0.66 \pm 0.76^{\mathrm{e}}$ & $154.13 \pm 6.56 \mathrm{abcd}$ & $104.53 \pm 3.02^{\text {cde }}$ \\
\hline \multirow[t]{5}{*}{30} & Control & $5.81 \pm 1.27 \mathrm{de}$ & $154.26 \pm 10.59 \mathrm{abc}$ & $120.30 \pm 6.90^{\mathrm{a}}$ \\
\hline & LT & $16.28 \pm 2.44^{\mathrm{abc}}$ & $135.80 \pm 19.57^{\mathrm{fg}}$ & $109.96 \pm 5.49^{b c}$ \\
\hline & HT & $13.69 \pm 3.13^{\mathrm{bcd}}$ & $137.6 \pm 19.31$ efg & $106.00 \pm 5.72$ bcde \\
\hline & LC & $13.35 \pm 5.74 \mathrm{bcd}$ & $140.43 \pm 21.90^{\text {cdefg }}$ & $109.80 \pm 5.81 \mathrm{bcd}$ \\
\hline & $\mathrm{HC}$ & $16.57 \pm 1.59^{\mathrm{ab}}$ & $143.10 \pm 15.48^{\text {bcdef }}$ & $110.73 \pm 6.37^{b}$ \\
\hline \multirow[t]{5}{*}{45} & Control & $7.85 \pm 1.65^{\text {cde }}$ & $151.42 \pm 9.55^{\mathrm{abcdef}}$ & $111.88 \pm 8.00^{\mathrm{b}}$ \\
\hline & LT & $11.48 \pm 6.08^{\mathrm{bcd}}$ & $133.35 \pm 22.18^{g}$ & $106.88 \pm 6.00^{\text {bcde }}$ \\
\hline & HT & $16.09 \pm 2.33 \mathrm{abc}$ & $137.82 \pm 18.25^{\text {defg }}$ & $102.97 \pm 6.85^{\mathrm{e}}$ \\
\hline & LC & $17.45 \pm 3.06^{\mathrm{ab}}$ & $138.24 \pm 19.2^{\text {cdefg }}$ & $106.57 \pm 6.56^{\text {bcde }}$ \\
\hline & $\mathrm{HC}$ & $23.44 \pm 3.70^{\mathrm{a}}$ & $136.08 \pm 19.08^{\mathrm{fg}}$ & $103.71 \pm 5.25^{\mathrm{de}}$ \\
\hline
\end{tabular}

Values shown are mean $( \pm$ SD) from $n=6$ rats/group at each timepoint. Values in each column followed by different letters are significantly different ( $\mathrm{p} \leq 0.05$ ). 


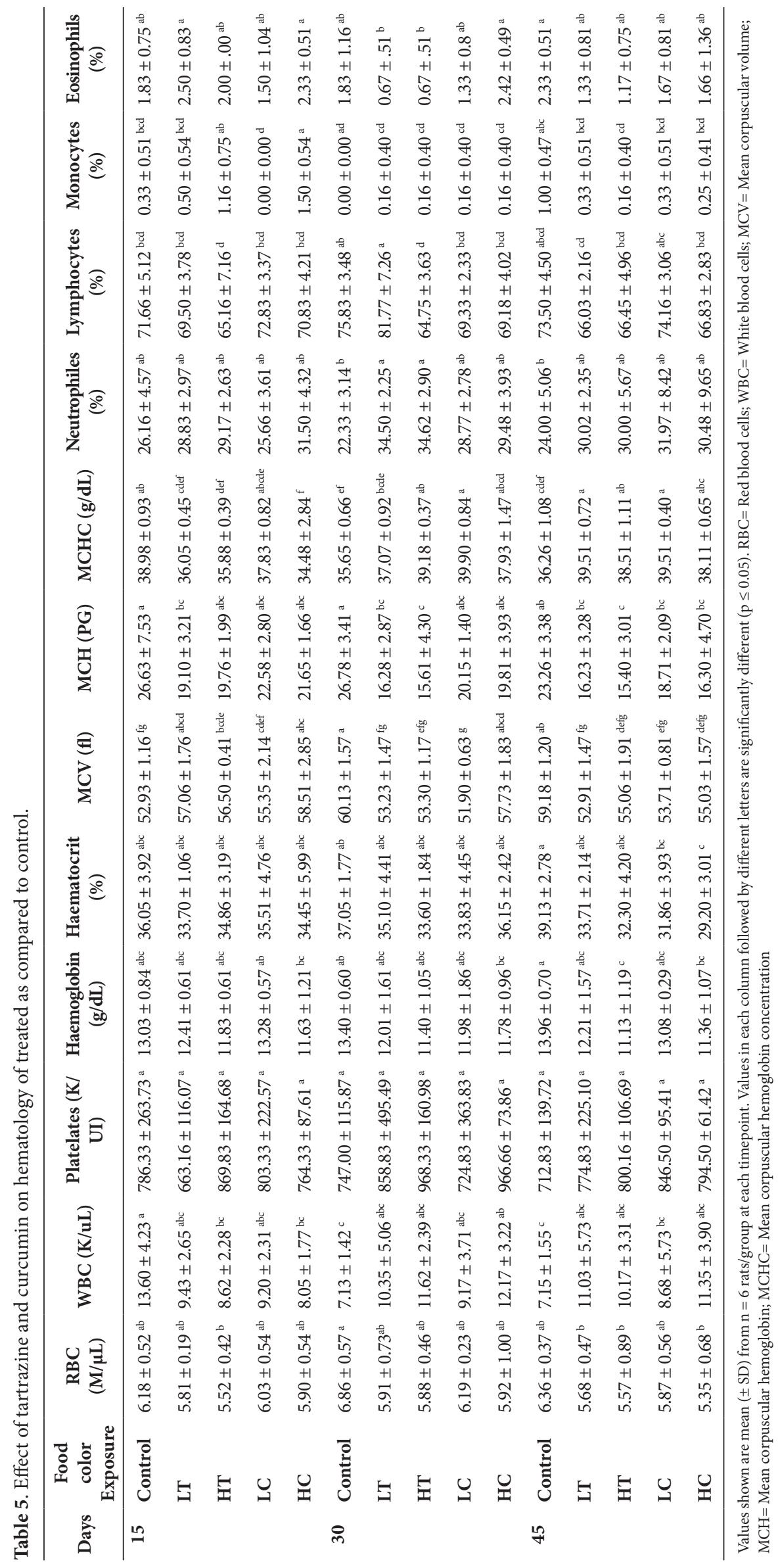


low in HT30 compared to control and LT30. There was significantly lower level of monocytes found in LC15 and HC15 in Table 5.

\subsection{Biochemical parameters}

\section{Liver enzymes}

There was considerable reduction in the level of ALP in HC15 while there was non-significant reduction were seen among treated groups of 30 and 45 days. On the other hand, high level of ALT noted after 45 days. A significant increment was observed in the concentration of AST in HT45, LC45 and HC45 group. Lower and higher doses of tartrazine and curcumin non-significantly decreased the level of $\mathrm{LDH}$ in the treated group of 15 days and increased in HC 45 (Table 6).

\section{Renal function markers}

BUN (blood urea nitrogen) level was increased in LT30, HT30 LT45, HT45, and HC45 respectively. Whereas significantly decreased in LC30 and HC30. ADI and higher doses of tartrazine and curcumin was non-significantly increased creatinine after 45 days. High level of creatinine was

Table 6. Effect of tartrazine and curcumin on hepatic function enzymes.

\begin{tabular}{|c|c|c|c|c|c|}
\hline Days & Food color Exposure & ALT (U/L) & $\operatorname{AST}(\mathrm{U} / \mathrm{L})$ & $\operatorname{ALP}(\mathrm{U} / \mathrm{L})$ & LDH (U/L) \\
\hline \multirow[t]{5}{*}{15} & Control & $33.33 \pm 9.75^{\mathrm{abc}}$ & $89.83 \pm 5.19^{c}$ & $40.05 \pm 5.45^{\mathrm{ab}}$ & $962.66 \pm 182.62^{a}$ \\
\hline & LT & $27.83 \pm 7.27 \mathrm{abc}$ & $80.16 \pm 8.03^{c}$ & $35.22 \pm 7.38^{\mathrm{abcd}}$ & $646.33 \pm 194.71^{a b}$ \\
\hline & HT & $24.5 \pm 3.14^{\mathrm{abc}}$ & $98.33 \pm 14.90^{b c}$ & $23.16 \pm 12.25^{\mathrm{abcd}}$ & $680.00 \pm 227.55^{\mathrm{ab}}$ \\
\hline & LC & $35.5 \pm 25.46^{a b c}$ & $86.66 \pm 14.47^{c}$ & $38.66 \pm 22.72^{a b c}$ & $727.00 \pm 116.37^{\mathrm{ab}}$ \\
\hline & $\mathrm{HC}$ & $40.66 \pm 21.74^{\mathrm{abc}}$ & $97.50 \pm 11.41^{b c}$ & $15.50 \pm 1.87^{\mathrm{cd}}$ & $539.83 \pm 163.41$ \\
\hline \multirow[t]{5}{*}{30} & Control & $24.83 \pm 4.66^{a b c}$ & $91.83 \pm 30.49^{c}$ & $45.8 \pm 23.79^{a}$ & $620.33 \pm 361.00^{a b}$ \\
\hline & LT & $18.33 \pm 3.44^{b c}$ & $92.5 \pm 23.60^{c}$ & $28.25 \pm 13.08^{a b c d}$ & $850.00 \pm 218.43^{a b}$ \\
\hline & HT & $15.66 \pm 3.38^{c}$ & $99.25 \pm 29.11 b c$ & $21.50 \pm 8.66^{\mathrm{abcd}}$ & $505.16 \pm 192.69^{b}$ \\
\hline & LC & $24.66 \pm 3.933^{a b c}$ & $77.00 \pm 29.61^{c}$ & $20.25 \pm 4.16^{\mathrm{abcd}}$ & $726.00 \pm 277.68^{a b}$ \\
\hline & HC & $39.66 \pm 25.76^{a b c}$ & $114.41 \pm 73.22^{\mathrm{abc}}$ & $24.5 \pm 5.07^{\mathrm{abcd}}$ & $749.50 \pm 152.05^{a b}$ \\
\hline \multirow[t]{5}{*}{45} & Control & $26.83 \pm 6.79^{a b c}$ & $89.00 \pm 27.01^{c}$ & $36.50 \pm 21.71^{\mathrm{abcd}}$ & $660.00 \pm 317.61$ \\
\hline & LT & $47.16 \pm 7.33^{a}$ & $105.50 \pm 34.14^{b c}$ & $21.25 \pm 9.98^{b c d}$ & $581.00 \pm 181.80^{a b}$ \\
\hline & HT & $48.5 \pm 9.58^{a}$ & $173.50 \pm 34.14^{\mathrm{a}}$ & $14.00 \pm 3.40^{\mathrm{d}}$ & $810.00 \pm 125.51$ \\
\hline & LC & $41.66 \pm 7.03^{a b}$ & $155.75 \pm 25.50^{\mathrm{ab}}$ & $24.00 \pm 10.41^{\mathrm{abcd}}$ & $814.33 \pm 93.70^{\mathrm{ab}}$ \\
\hline & $\mathrm{HC}$ & $46.5 \pm 6.97^{\mathrm{a}}$ & $152.25 \pm 28.59^{a b}$ & $17.00 \pm 6.81^{\mathrm{bcd}}$ & $940.00 \pm 169.24^{\mathrm{a}}$ \\
\hline
\end{tabular}

Values shown are mean $( \pm S D)$ from $n=6$ rats/group at each timepoint. Values in each column followed by different letters are significantly different ( $\mathrm{p} \leq 0.05)$. ALT $=\mathrm{Alanine}$ transaminase; AST $=$ Aspartate Transaminase; ALP $=$ Alkaline Phosphatase; $\mathrm{LDH}=$ Lactate Dehydrogenase

Table 7. Effect of tartrazine and curcumin on renal function marker

\begin{tabular}{|c|c|c|c|c|c|c|c|c|c|}
\hline Days & $\begin{array}{c}\text { Food } \\
\text { color } \\
\text { Exposure }\end{array}$ & BUN (mg/dL) & $\begin{array}{c}\text { CREATINE } \\
(\mathrm{mg} / \mathrm{dL})\end{array}$ & $\begin{array}{l}\text { URIC ACID } \\
(\mathrm{mg} / \mathrm{dL})\end{array}$ & $\begin{array}{l}\text { ALBUMIN } \\
(\mathrm{g} / \mathrm{dL})\end{array}$ & $\begin{array}{c}\text { BILIRUBIN } \\
(\mathrm{mg} / \mathrm{dL})\end{array}$ & $\begin{array}{c}\text { GLOBULIN } \\
(\mathrm{g} / \mathrm{dL})\end{array}$ & A/G RATIO & $\begin{array}{c}\text { TOTAL } \\
\text { PROTEIN } \\
(\mathrm{g} / \mathrm{dL})\end{array}$ \\
\hline \multirow[t]{5}{*}{15} & Control & $46.81 \pm 5.58$ abcd & $0.30 \pm 0.02^{\mathrm{d}}$ & $1.65 \pm 0.25^{b}$ & $4.30 \pm 1.00^{\text {bcd }}$ & $0.25 \mathrm{v} \pm 0.04^{\mathrm{c}}$ & $3.65 \pm 0.37 \mathrm{bc}$ & $1.01 \pm 0.12^{b}$ & $8.36 \pm 0.44^{b}$ \\
\hline & LT & $42.0 \pm 17.22^{\mathrm{bcd}}$ & $0.46 \pm 0.05^{\mathrm{cd}}$ & $0.42 \pm 0.17^{\mathrm{cd}}$ & $3.42 \pm 0.68^{\text {def }}$ & $0.15 \pm 0.08^{c}$ & $3.60 \pm 0.33^{b c}$ & $1.12 \pm 0.04^{\mathrm{ab}}$ & $7.50 \pm 0.48 \mathrm{abcd}$ \\
\hline & HT & $59.25 \pm 5.68 \mathrm{abc}$ & $0.71 \pm 0.37^{\mathrm{abcd}}$ & $0.75 \pm 0.26^{\mathrm{bcd}}$ & $3.22 \pm 0.82^{\text {def }}$ & $0.17 \pm 0.04^{c}$ & $2.90 \pm 0.38^{c}$ & $1.43 \pm 0.33^{\mathrm{ab}}$ & $7.72 \pm 0.73 \mathrm{abc}$ \\
\hline & LC & $40.5 \pm 15.41^{\mathrm{bcd}}$ & $0.60 \pm 0.18^{\mathrm{bcd}}$ & $0.22 \pm 0.10^{\mathrm{d}}$ & $4.56 \pm 0.24^{\mathrm{bc}}$ & $0.15 \pm 0.05^{c}$ & $4.05 \pm 0.61^{b}$ & $1.09 \pm 0.12^{b}$ & $8.35 \pm 0.86^{\mathrm{ab}}$ \\
\hline & $\mathrm{HC}$ & $29.25 \pm 3.79^{d}$ & $0.43 \pm 0.05^{\mathrm{cd}}$ & $0.55 \pm 0.17^{\mathrm{bcd}}$ & $3.46 \pm 0.46^{\text {cdef }}$ & $0.10 \pm 0.00^{c}$ & $3.92 \pm 0.52^{b}$ & $1.47 \pm 0.68^{\mathrm{ab}}$ & $7.30 \pm 0.97 \mathrm{abcd}$ \\
\hline \multirow[t]{5}{*}{30} & Control & $45.66 \pm 10.21^{\mathrm{abcd}}$ & $0.92 \pm 0.47^{\mathrm{abc}}$ & $3.07 \pm 0.86^{\mathrm{a}}$ & $4.30 \pm 1.00^{\text {bcde }}$ & $0.67 \pm 0.29^{\mathrm{ab}}$ & $4.22 \pm 0.44^{\mathrm{b}}$ & $1.18 \pm 0.40^{\mathrm{ab}}$ & $8.26 \pm 1.28^{a b}$ \\
\hline & LT & $61.25 \pm 10.21 \mathrm{ab}$ & $1.13 \pm 0.31^{\mathrm{ab}}$ & $1.30 \pm 0.93^{\text {bcd }}$ & $3.41 \pm 0.49^{\mathrm{def}}$ & $0.37 \pm 0.31^{b c}$ & $3.48 \pm 0.44^{\mathrm{bc}}$ & $1.30 \pm 0.23^{\mathrm{ab}}$ & $8.65 \pm 0.63^{\mathrm{a}}$ \\
\hline & HT & $59.41 \pm 14.15^{\mathrm{abc}}$ & $0.39 \pm 0.04^{\mathrm{cd}}$ & $1.60 \pm 0.57^{b}$ & $3.21 \pm 0.87^{\text {ef }}$ & $0.17 \pm 0.10^{c}$ & $3.28 \pm .88^{\mathrm{bc}}$ & $1.75 \pm 0.22^{\mathrm{a}}$ & $6.48 \pm 0.99^{\mathrm{cd}}$ \\
\hline & LC & $33.00 \pm 9.82^{d}$ & $1.18 \pm 0.48^{\mathrm{a}}$ & $1.03 \pm 0.57^{\mathrm{bcd}}$ & $4.10 \pm 0.23^{\text {bcde }}$ & $0.25 \pm 0.05^{\mathrm{c}}$ & $3.55 \pm 0.70^{\mathrm{bc}}$ & $1.22 \pm 0.23^{\mathrm{ab}}$ & $6.83 \pm 0.60^{\mathrm{cd}}$ \\
\hline & $\mathrm{HC}$ & $34.25 \pm 6.19^{c d}$ & $0.68 \pm 0.48$ abcd & $1.15 \pm 0.59^{\text {bcd }}$ & $4.50 \pm 0.20^{\text {bcd }}$ & $0.20 \pm 0.06^{\mathrm{c}}$ & $3.37 \pm .42^{\mathrm{bc}}$ & $1.53 \pm 0.39^{\mathrm{ab}}$ & $6.60 \pm 0.31^{\mathrm{cd}}$ \\
\hline \multirow[t]{5}{*}{45} & Control & $48.71 \pm 12.48$ abcd & $0.32 \pm 0.19^{d}$ & $3.22 \pm 1.11^{\mathrm{a}}$ & $5.00 \pm 0.36 \mathrm{ab}$ & $0.73 \pm 0.36^{\mathrm{a}}$ & $4.15 \pm 0.37^{b}$ & $1.09 \pm 0.13^{b}$ & $8.50 \pm 0.73^{\mathrm{a}}$ \\
\hline & LT & $63.75 \pm 17.02 \mathrm{ab}$ & $0.55 \pm 0.15^{\mathrm{cd}}$ & $1.42 \pm 0.35^{b c}$ & $3.20 \pm 0.18^{\text {ef }}$ & $0.17 \pm 0.04^{c}$ & $3.58 \pm 0.31^{b c}$ & $1.31 \pm 0.11^{\mathrm{ab}}$ & $7.00 \pm 0.55^{\text {bcd }}$ \\
\hline & HT & $70.00 \pm 12.55^{\mathrm{a}}$ & $0.79 \pm 0.15^{\mathrm{abcd}}$ & $1.39 \pm 0.36^{b c}$ & $2.98 \pm 0.26^{\mathrm{f}}$ & $0.17 \pm 0.04^{c}$ & $3.45 \pm 0.33^{b c}$ & $1.27 \pm 0.52^{\mathrm{ab}}$ & $6.20 \pm 0.17^{\mathrm{d}}$ \\
\hline & LC & $48.75 \pm 7.13^{\mathrm{abcd}}$ & $0.55 \pm 0.12^{\mathrm{cd}}$ & $0.35 \pm 0.10^{\mathrm{cd}}$ & $6.02 \pm 0.45^{\mathrm{a}}$ & $0.20 \pm 0.07^{c}$ & $3.45 \pm 0.33^{b c}$ & $1.14 \pm 0.07^{\mathrm{ab}}$ & $6.72 \pm 0.57^{\mathrm{cd}}$ \\
\hline & $\mathrm{HC}$ & $70.25 \pm 17.69^{a}$ & $0.52 \pm 0.10^{\mathrm{cd}}$ & $1.00 \pm 0.50^{\mathrm{bcd}}$ & $3.63 \pm 0.69^{\text {cdef }}$ & $0.22 \pm 0.04^{c}$ & $5.25 \pm 034^{\mathrm{a}}$ & $1.44 \pm 0.36^{\mathrm{ab}}$ & $6.43 \pm 0.27^{\mathrm{cd}}$ \\
\hline
\end{tabular}

Values shown are mean $( \pm \mathrm{D})$ from $\mathrm{n}=6$ rats/group at each timepoint. Values in each column followed by different letters are significantly different ( $\mathrm{p} \leq 0.05)$. BUN $=$ Blood Urea Nitrogen. 
Table 8. Effect of tartrazine and curcumin on blood glucose and cholesterol.

\begin{tabular}{|c|c|c|c|c|}
\hline Days & Food color Exposure & GLUCOSE (mg/dL) & CHOLESTROL (mg/dL) & TRIGLYCERIDES (mg/dL) \\
\hline \multirow[t]{5}{*}{15} & Control & $84.25 \pm 6.91^{\text {cde }}$ & $42.75 \pm 6.95^{\mathrm{ab}}$ & $103.83 \pm 87.08^{\mathrm{bcd}}$ \\
\hline & LT & $83.00 \pm 9.44^{\mathrm{cde}}$ & $28.25 \pm 6.72^{c}$ & $41.66 \pm 6.40^{\mathrm{ef}}$ \\
\hline & HT & $67.75 \pm 14.42^{\text {ef }}$ & $30.00 \pm 5.10^{\mathrm{abc}}$ & $36.33 \pm 24.99^{e f}$ \\
\hline & LC & $71.75 \pm 5.55^{\text {def }}$ & $30.00 \pm 3.86^{b c}$ & $16.50 \pm 4.84^{\mathrm{f}}$ \\
\hline & $\mathrm{HC}$ & $70.95 \pm 10.23^{\text {def }}$ & $31.25 \pm 6.58^{\mathrm{bc}}$ & $21.50 \pm 2.73^{\text {ef }}$ \\
\hline \multirow[t]{5}{*}{30} & Control & $93.00 \pm 14.51^{\mathrm{bcd}}$ & $50.00 \pm 9.08^{\mathrm{a}}$ & $94.67 \pm 40.66^{\text {cdef }}$ \\
\hline & LT & $89.25 \pm 8.02^{\text {cde }}$ & $39.78 \pm 2.27 \mathrm{abc}$ & $80.17 \pm 42.57$ def \\
\hline & HT & $56.75 \pm 19.58^{\mathrm{f}}$ & $37.85 \pm 5.25 \mathrm{abc}$ & $81.00 \pm 44.06$ def \\
\hline & LC & $85.00 \pm 4.52^{\mathrm{cde}}$ & $36.75 \pm 6.55 \mathrm{abc}$ & $41.50 \pm 12.011^{\text {ef }}$ \\
\hline & $\mathrm{HC}$ & $73.25 \pm 7.64 \mathrm{def}$ & $40.63 \pm 7.25^{\mathrm{abc}}$ & $57.83 \pm 13.32 \mathrm{def}$ \\
\hline \multirow[t]{5}{*}{45} & Control & $93.00 \pm 4.33^{\mathrm{bcd}}$ & $42.50 \pm 11.91 \mathrm{ab}$ & $131.83 \pm 38.54 \mathrm{bcd}$ \\
\hline & LT & $104.00 \pm 19.06^{a b c}$ & $33.75 \pm 3.51^{b c}$ & $188.33 \pm 42.01^{a b}$ \\
\hline & HT & $122.50 \pm 18.22^{a}$ & $30.00 \pm 4.34^{b c}$ & $229.50 \pm 57.80^{a}$ \\
\hline & LC & $76.75 \pm 10.10^{\text {def }}$ & $28.50 \pm 10.17^{c}$ & $179.33 \pm 35.36^{a b c}$ \\
\hline & $\mathrm{HC}$ & $115.50 \pm 10.38^{a b}$ & $30.00 \pm 6.90^{\mathrm{bc}}$ & $244.16 \pm 66.89^{\mathrm{a}}$ \\
\hline
\end{tabular}

Values shown are mean $( \pm S D)$ from $n=6$ rats/group at each timepoint. Values in each column followed by different letters are significantly different ( $\mathrm{p} \leq 0.05$ ).

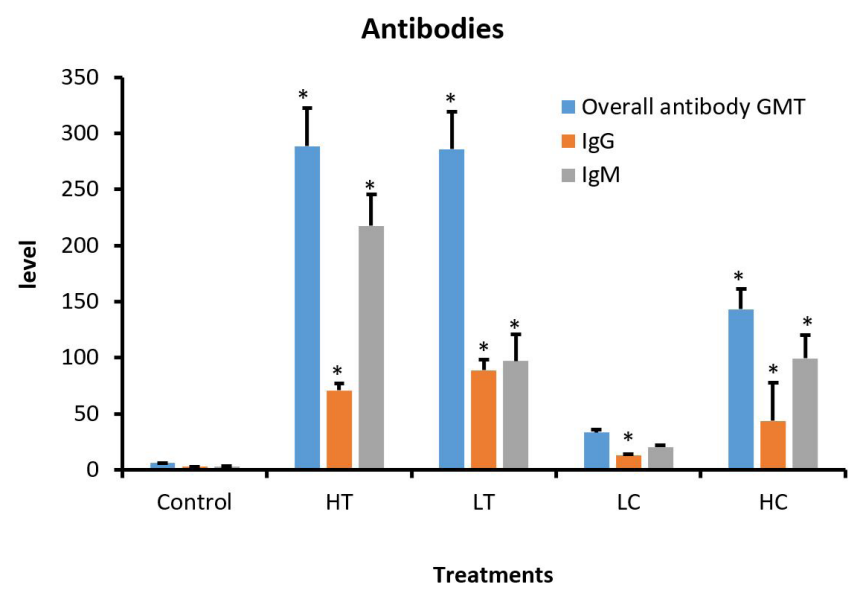

Figure 2. Effect of tartrazine and curcumin on IgG and IgM of control animal as compared to control after 45 days of treatment. Treatments having the asterisk $\left(^{*}\right)$ are significantly different from control group.

noted in HT15 and HT45 whereas creatinine was decreased in HT30 (Table 7).

Furthermore, reduction was noted in the level of globulin after 30 days in all treatments (Table 7). Following 30 and 45 days, uric acid was radically low in treated clusters (LT vs HT, LC vs HC were found non-significantly difference).

High tartrazine supplementation for 45 days had significantly exhibited lower level of albumin. Considerable lower level of bilirubin was seen in HT30, LC30, Hc30 and all treatment groups of 45 days compared to control. Additionally, non-significant reduction was seen in globulin of all treated groups of 30 days, HT15, LT45, HT45 and LC45.

Blood glucose and cholesterol

The level of glucose was decreased in HT30 whereas, significantly increased in HT45 and HC45 groups respectively. There was a significant $(\mathrm{P} \leq 0.05)$ lower concentration of cholesterol in LT15 and LC45 while non-significant reduction were seen among all treatments. Triglycerides was significantly high in treated group after 15 days, HT45 and HC45 respectively compared to control (Table 8).

\subsection{Immunological parameters}

Figure 2 shows changes in the IgG, IgM and overall antibody GMT. Changes were noted only in tartrazine and curcumin treated groups after 45 days. IgG, IgM, and overall antibody GMT were considerably decreased in treated groups.

\section{Discussion}

In this current study, the effect of food colors assessments on biochemical, hematological and immunological parameters in adult female rats treated with lower (ADI) and high (10 time of ADI) dose of tartrazine and curcumin for 15, 30 and 45 days. Purpose of choosing high dose was made to evaluate the safest level that means how long we can use natural and artificial food colors without any problem.

The gain of b.w was about 20 to $25 \%$ from the initial b.w. Results were in accord with Sharma (2015) who described an increment in the b.w over $20 \%$ above the mean b.w was considered as obesity. While, El Golli et al. (2016) described opposite result that tartrazinetreated rats indicated a significant decrease in b.w at the end of study. 
Changes in b.w are considered a toxicity indicator. Thus, the difference in b.w reported in many studies may represent the first marker of adverse effect. Moreover, El-Wahab \& Moram (2012) reported that synthetic food colorants might bind to the bacterial cell surface in the rat's intestine, leading to a reduction in the numeral of active bacterial cells and an inhibition of the food absorption capacity at the intestinal surface, leading to a BW decrease or increase

Another possible reason of increment in b.w was that after oral ingestion of food color or parenteral administration, compounds of color may reaches into the gut promptly or through the bile. It could subject to the digestive enzymes, working of gastric acids as well as microbiota (Amin et al., 2010).

Water and feed intake were decreased after 30 and 45 days. Findings are similar with Mehedi et al. (2009) who confirmed that feed ingestion was reduced experimental animals, whereas for water intake showed different result from the current study. The low in food and water intake was possibly due to the incorporation of tartrazine and curcumin. It was hypothesized that the digestion of diet may be inhibited in a certain manner by the supplement of synthetic colorant or synthetic flavors. Changes in food intake were not parallel to the growth, feed efficiency for the different colorants (El-Wahab \& Moram, 2012). This impact lessened the totals of the active bacterial cells in the gut, in which the decreased population of intestine microbiota hindered the food absorption capability of the intestinal surface.

Findings from the proximate analysis are related with conclusion of Oluwaniyi et al. (2009) who described that proximate composition of the synthetic colorants had higher ash contents (79.50-82.50\%) in contrast to the natural colorants (15.75-22.28\%). The reverse was the case for carbohydrate and fiber contents of the colorants. The synthetic colorants had less carbohydrate and fiber content (between 2.39 and 3.99\%) whereas the natural colorants had values between 44.20 and $68.69 \%$. Alternatively, Chattopadhyay et al. (2011) reported that curcumin had crude protein $(6.3 \%)$, fat content $(5.1 \%)$, minerals (3.5\%), carbohydrates (69.4\%) and moisture (13.1\%). Differences in the result might be due to the commercially available brands of curcumin. By adding these both food colors into the feed of rat, low and high doses of tartrazine and curcumin change the composition of the animal diet. Sufficient data is not available to support the current results of compositional analysis of curcumin and tartrazine containing feed samples.

Various qualitative and quantitative phytochemical tests were performed for selected secondary metabolites. In the current study, an attempt was made to investigate phytochemical evaluation of feed which contains lower and higher doses of tartrazine and curcumin. Crude extract (Aqueous, ethanolic and methanolic) of the control and feed with tartrazine and curcumin were taken for the study. The phytochemical screening was done for the 5 feeds samples.

Many phytochemical constituents were significantly altered the physiological parameter of the kidney. The saponin significantly alters the level of urea, uric acid, creatinine and plasma protein by changing the physiology of kidney. The extracts of saponin did not indicate any harmful result on the organs despite its positive therapeutic actions on infections caused by E. coli and Salmonella typhi (Ajibade \& Famurewa, 2012). Flavonoids have a protective effect on the kidney. The extracting solvents (ethanol, methanol, water, hexane, ethyl-acetate, and acetone) could affect phytochemicals content and antioxidant activities (Qiang, 2015). The presence and absence of phytochemicals in non-detectable quantity or non-solubility in the solvents employed for the studied food colors and feed. Differences in the presence or absence of compounds in the current study and earlier reports on the same species or related species may be due to the genetic component or geographical/biotic environment or both (Saivenkatesh \& Murthy, 2016).

The high amount of carbohydrates in ethanolic and methanolic extract of high curcumin (HC) feed was $3691 \mathrm{mg} / \mathrm{dL}$, flavonoids were $404 \mu \mathrm{g} / \mathrm{mL}$ in ethanolic extract of $\mathrm{HC}$, phenols were $114 \mu \mathrm{g} / \mathrm{mL}$ in methanolic extracts of $\mathrm{HC}$, alkaloids were $228 \mathrm{mg} / \mathrm{dL}$ and tannins were $295 \mathrm{~g} / \mathrm{mL}$ in methanolic extract of low curcumin feed described in Table 4. Current results are in accordance with that of Saxena \& Rajeshwari (2012) who recorded ten phytochemicals (Carbohydrate, proteins, starch, amino acids, steroid, glycoside, flavonoid, alkaloid, tannin, and saponin) from methanolic extracts of rhizomes of Curcumin. The primary phytochemical evaluation of the methanolic extract indicated the existence of tannins, alkaloids, saponins, flavonoids, terpenoids and cardiac glycosides reported by Rajesh et al. (2013). Swadhini et al. (2011) documented six phytochemicals viz alkaloids, flavonoids, tannin, saponins, cardiac glycosides and phenol from aqueous extract of turmeric. Agreeing to Paliwal et al. (2011), Curcuma caesia extracts have major components for example alkaloids, steroids, phenolics and tannins.

The RBCs is the very effective as raw data for the estimation of the erythrocytes indices MCV and MCH. Reduced RBC is usually observed in anemia of any cause (AL-Shinnawy, 2009). According to Stevens et al. (2013), iron holds essential roles in most biochemical reactions in the body. Tartrazine has led to reductions in hemoglobin and hematocrit levels which cause anemia in rats (Sharma et al., 2009). Thus, it is considered that consumption of food containing tartrazine for 45 days may cause iron deficiency and anemia. Furthermore, curcumin was also found to be an active iron chelator in vivo and induced a state of overt iron deficiency anemia in mice fed with diets poor in iron (Sharma et al., 2009).

Flavonoids are responsible in changing the concentration of mean corpuscular hemoglobin and their occurrence in all color feed causes the alteration after 15, 30 and 45 days of treatment with tartrazine and curcumin (Jabeen et al., 2013). According to Stock \& Hoffman (2000) flavonoid could cause the increase concentration of mean corpuscular hemoglobin concentration and its presence in food color containing feed might responsible for the increase concentration of $\mathrm{MCHC}$ in all treated rats after 30 and 45 days.

White blood cells (WBC) are an essential pillar for host defense system and responsible for safety against bacteria, fungi, invading parasites and viruses (Stock \& Hoffman, 2000). Elevated WBC value designates bone marrow distortions and inflammation whereas less WBC count discloses leucopenia 
or medications that defeat or weaken the immune system or deplete the bone marrow (Stock \& Hoffman, 2000). Alkaloid, glycoside, saponin and tannin in food colors cause an increasing number of white blood cell (Adedapo et al., 2007). The existence of this component in colored feed might be accountable for the increased count of WBC in the treatment group of experimental subjects. Current findings are concordance with Montejo et al. (2015) and Hashem et al. (2010) who resulted that tartrazine and metanil yellow induced a significant decrease in glucose, alkaline phosphatase (ALP), and total cholesterol levels. Decreased level of glucose caused hypoglycemia and this might be justified by changes in blood sugar level caused by foreign compounds (Timbrell, 2009).

In this study, fluctuation was seen in the level of ALT (48 U) and AST (173U) in HT group. The fluctuation in liver enzymes indicates the damage produced in hepatocytes, which consequently causes the release of intracellular enzymes in the blood. Therefore, increased plasma levels of AST and ALT suggested damage of both hepatic cellular and mitochondrial membranes (El-Wahab \& Moram, 2012). Likewise, the rise in plasma LDH could be attributed to cell damage induced by tartrazine and curcumin. Reduction in LDH is may be due to body breaks down sugar for use as energy in cells. These finding are similar with Amin et al. (2010) who indicated rise in serum ALT, AST, and ALP actions using $500 \mathrm{mg}$ of tartrazine $/ \mathrm{kg}$ of BW/day in young rats, during a 30 days period.

Recently, Khayyat et al. (2017) mentioned that consumption of tartrazine considerably higher the activity of serum ALP, AST, and ALT. The liver damage would release a higher than normal level of intracellular enzymes in the blood. ALP has several physiological functions in bone cells, it splits inorganic phosphates from organic phosphate which is a potent inhibitor of mineralization (Charles et al., 1992). The results of the present study showed significant increase in ALP activity for tartrazine may be attributed to the defect in liver function (Hayes, 1994). The liver synthesizes proteins, among which is albumin. Findings are similar with El Golli et al. (2016) who concluded that serum albumin was significantly reduced after tartrazine treatment, as well as serum total protein and globulin. Although findings of the study is in contrast with the Amin et al. (2010) and El Wahab \& Moram (2012) who reported significant increase in serum albumin concentration. Reason might be that tartrazine and curcumin exposure leads to deleterious effects on the synthetic function of treated rats' livers. Proteins are the fundamental components of enzymes, hormones and antibodies that are necessary for the proper functioning of an organism, increased release of enzymes by the damaged tissues and the antibodies to counteract the dye might be the cause of increase in serum protein (Sharma et al., 2005).

In this study results revealed significant decrease in the cholesterol level after ADI and higher doses of tartrazine and curcumin at 15, 30 and 45 days were in accord with outcomes of Amin et al. (2010) recorded depletion of cholesterol in rats administered 15 and $500 \mathrm{mg}$ of tartrazine $/ \mathrm{kg}$ of BW/ day. Cholesterol is a lipid family of sterols produced from Acetyl-CoA. The TC content depends on the balance between cholesterol formed in the body and the rate absorbed from the diet. Since the liver is the main site of production of cholesterol (20-25\% of daily production), the deviation of its normal in the blood is considered to be another marker of liver damage. The increment of triglycerides was observed in LT, HT, LC and HC 45. The increase in TG levels observed in the current study is consistent with Amin et al. (2010) who described that the surge in TG readings might be caused by an alteration of the activity of hepatic lipase, responsible for the catabolism of TGs (Ashour \& Abdelaziz, 2009). Thus, in the case of liver impairment, the lipase activity will be decreased resulting in hypertriglyceridemia. The reduction in plasma cholesterol level obtained is similar well with Ashour \& Abdelaziz (2009) who found a significant decrease in serum total cholesterol and triglycerides level.

Current study showed that tartrazine induced a significant increase in plasma creatinine in HT 15, HT45 but decrease in HT 30. These results are partially consistent with that of El-Wahab \& Moram (2012) who found elevated creatinine in tartrazine and brilliant blue treated rats. Increased creatinine level is observed when renal function is disturbed. Ali et al. (2016) also shown that tartrazine induces a significant increased level of creatinine and urea in the treated animal model. The substantial increment in plasma urea and creatinine level indicating the development of an acute renal dysfunction in rats. Study showed reduced uric acid which are in contrast with the result of Mehedi et al. (2009) who concluded that regular ingestion of tartrazine for thirteen weeks by male and female ( $2.5 \%$ dosage group) exhibited a major surge in uric acid level.

Neutrophiles and monocytes play an important role as a phagocytic cell. It is now well established that resistance to infection depends principally upon interaction of phagocytes and humoral factors (antibody and complement). Thus, the integrity of the cellular immune response might be affected by reduced level of neutrophiles and monocytes percent reported in rats (Yoshitake \& Sato, 1992). On the other hand, number of lymphocytes is high in viral infection while in a bacterial and allergic reaction, it goes down (Ansari-Lari et al., 2003). Alkaloids are liable to modify the lymphocyte and alter the numbers of lymphocyte in rats of all groups of 30 and 45 days (Vjibade \& Egbebi, 2011). The results of the clinical trials also showed similar changes as reported by Milagres et al. (2020).

\subsection{Antibody response to sheep $R B C s$}

Current study demonstrated that higher level of IgG, IgM and overall antibody GMT values in low tartrazine, high tartrazine, and high curcumin group was observed at 45 days compared to control. On the other side, low curcumin colorant showed significant lower level of IgG. Finding are in accordance with Ronis \& Cunny (2001) who resulted that the reduction of the titles in IgG anti-BSA reported in male mice treated with $0.45 \%$ and $1 \%$ of Tartrazine could affect the integrity of the humoral immune response. The high and low level of IgG differences may be due to those related to the animal sex there are a number of documented differences concerning the disposition and toxicity of foreign compounds which are related to the sex of the animal (Sex differences in metabolism are due to the influence of hormones and genetic factors. Many of the gender differences are developed during puberty for the animal and are influenced by sex hormone 
concentrations or indirect effects of growth hormone (El-Malky et al., 2014). Findings of present study were related to El Golli et al. (2016) who indicated that sub chronic exposure to the tartrazine has impaired the changes in blood parameters, immune response, hepatic and renal functions. Soltan \& Shehata (2012) also described that the higher dose of synthetic food colorants leads to rise the number of $\mathrm{WBC}$ as the result of the response of the immune system towards inflammation. Himri et al. (2011) further explained that consumption of 10 time high doses of sunset yellow, amaranth and curcumin can cause negative effects on the cellular but not humoral immune response. The free radicals may affect the level of antibodies and produce an imbalance between pro and antioxidants and weakens the cellular defense and might cause oxidative stress related disorders (El Golli et al., 2016; Demirkol et al., 2012). The individual response varies not only according to dose, gender, nutritional status, and genetic factors but also according to long term exposure to low doses (Sasaki et al., 2002).

\section{Conclusion}

Artificial and natural colors such as tartrazine and curcumin can influence negatively and alters the biochemical marker, hematological parameters and immunological status. Tartrazine and curcumin alter the renal and hepatic functions not only at high doses but also at lower doses. Effects of artificial and natural food colors become riskier at higher doses. Moreover, when added into the feed or food at recommended and higher dosage, it changed the nutritional composition of feed and also enhanced the few phytochemicals content which are not healthy i.e. tannins and CHO. Positive effects of curcumin is much higher than negative effects but it does not necessarily mean that the risk ratio of curcumin is shifted towards the benefit; it may just indicate that there are more researchers evaluating the beneficial effects of curcumin than evaluating its toxicity. It is the opinion of the authors that future research is needed to establish the benefit-risk profile of curcumin. In the meantime, it is important to acknowledge the negative properties of curcumin so that the use of curcumin is not based on unbalanced information.

Thus, it is important to understand the upcoming consequences by the use of food color in food products by worldwide food industries. Whereas, it's necessary to fruitfully apply coloring agents during processing and formation of foodstuffs.

\section{Acknowledgements}

Special thanks to the Faculty of Medicine and Health Science library for providing lab facility material, data and research papers to complete this manuscript. Also thanks to Mansoor Ahmad Khuhro for providing help in statistical analysis.

\section{References}

Adedapo, A. A., Abatan, M. O., \& Olorunsogo, O. O. (2007). Effects of some plants of the spurge family on the hematological and biochemical parameters of rats. Veterinarski Arhiv, 77(1), 29-38.

Ajibade, V. A., \& Famurewa, O. (2012). Histopathological and toxicological effects of crude saponin extract from Phyllanthus niruri, L (Syn. P. franternus. Webster) on organs in animal studies. Global Journal of Medical Research, 12(1), 31-37.
Ali, F. A., Abdelgayed, S. S., El-Tawil, S. O., \& Bakeer, M. A. (2016). Toxicological and histopathological studies on the effect of tartrazine in male albino rats. International Journal of Biological, Biomolecular, Agriculture, Food and Biotechnological Engineering, 10(8), 473-478.

AL-Shinnawy, M. (2009). Physiological effect of a food additive on some hematological and biochemical parameters of male albino rats. Egyptian Academic. Journal of Biological Sciences, 2(1), 143-151. http://dx.doi.org/10.21608/eajbsa.2009.15511.

American Association of Cereal Chemists - AACC. (2000). Approved methods of the American Association of Cereal Chemists (10th ed.). St. Paul: AACC.

Amin, K. A., Abdel Hameid, H. 2nd, \& Abd Elsttar, A. H. (2010). Effect of food azo dyes tartrazine and carmoisine on biochemical parameters related to renal, hepatic function and oxidative stress biomarkers in young male rats. Food and Chemical Toxicology, 48(10), 2994-2999. http://dx.doi.org/10.1016/j.fct.2010.07.039. PMid:20678534.

Amir, M. A., Khan, A., Mujeeb, M., Ahmad, M. A., \& Siddiqui, N. A. (2011). Phytochemical screening and in vitro antioxidant activity of Jawarish Aml a poly herbal formulation. Pharmacognosy Journal, 3(26), 54-60. http://dx.doi.org/10.5530/pj.2011.26.10.

Ansari-Lari, M. A., Kickler, T. S., \& Borowitz, M. J. (2003). Immature granulocyte measurement using the Sysmex XE-2100. Relationship to infection and sepsis. American Journal of Clinical Pathology, 120(5), 795-799. http://dx.doi.org/10.1309/LT30BV9UJJV9CFHQ. PMid:14608908.

Ashour, A. A., \& Abdelaziz, I. (2009). Role of fast green on the blood of rats and the therapeutic action of vitamins $\mathrm{C}$ or $\mathrm{E}$. International Journal of Integrative Biology, 6(1), 6-11.

Balabhaskar, R., \& Vijayalakshmi, K. (2015). Preliminary phytochemical and pharmacognostic analysis of Bauhinia tomentosa. Journal of Chemical and Pharmaceutical Research, 7(4), 271-277.

Ben, I. O., Woode, E., Abotsi, W. K. M., \& Gyasi, E. B. (2013). Preliminary phytochemical screening and in vitro antioxidant properties of Trichilia monadelpha (Thonn) J.J. de wild (Meliaceae). Journal of Medical and Biomedical Sciences, 2(2), 6-15.

Charles, P., Hasling, C., Risteli, L., Risteli, J., Mosekilde, L., \& Eriksen, E. F. (1992). Assessment of bone formation by biochemical markers in metabolic bone disease: Separation between osteopathic activity at the cell and tissue level. Calcified Tissue International, 51(6), 406-411. http://dx.doi.org/10.1007/BF00296671 PMid:1451006.

Chattopadhyay, I., Kaushik, B., Bandyopadhyay, U., \& Banerjee, R. (2011). Turmeric and curcumin: biological actions (pp. 2081-2087). Lucknow, India: Industrial Toxicological Research Center.

Chung, K. T., Stevens, J. R. E. Jr, \& Cerniglia, C. E. (1992). The reduction of dyes by the intestinal microflora. Critical Reviews in Microbiology, 18(3), 175-190. http://dx.doi.org/10.3109/10408419209114557 PMid:1554423.

Demirkol, O., Zhang, X., \& Ercal, N. (2012). Oxidative effects of Tartrazine (CAS No. 1934-21-0) and New Coccin (CAS No. 261182-7) azo dyes on CHO cells. Journal für Verbraucherschutz und Lebensmittelsicherheit, 7(3), 229-236. http://dx.doi.org/10.1007/ s00003-012-0782-z.

El Golli, N., Bini-Dhouib, I., Jrad, A., Boudali, I., Nasri, B., Belhadjhmida, N., \& El Fazaa, S. (2016). Toxicity induced after subchronic administration of the synthetic food dye tartrazine in adult rats, role of oxidative stress. Recent Advances in Biology and Medicine, 2, 21-28. http://dx.doi.org/10.18639/RABM.2016.02.284474. 
El-Malky, W. A., Khiralla, G. M., \& Salem, S. A. (2014). Nutritional study of some food coloring agents on experimental rats. International Journal of Nutrition and Food Sciences, 3(6), 538-544.

El-Wahab, H. M. F., \& Moram, G. S. (2012). Toxic effects of some synthetic food colorants and/or flavor additives on male rats. Toxicology and Industrial Health, 29(2), 224-232. http://dx.doi. org/10.1177/0748233711433935. PMid:22317828.

Export-Import Bank of India - EIBI. (2010). The EU market for natural colours, flavours and thickeners. Agricultural Export Advantage, 24(5), 4-8.

Ghongade, R. (2013). Phytochemical analysis of Citrus karna fruit. International Journal of Pharma and Bio Sciences, 4(2), 1162-1167.

Harsha, N., Sridevi, V., Lakshmi, M. V. V., Rani, K., \& Vani, N. D. S. (2013). Phytochemical analysis of some selected spices. International Journal of Innovative Research in Science Enineering and Technology, 2, 6618-6621.

Hashem, M. M., Atta, A. H., Arbid, M. S., Nada, S. A., \& Asaad, G. F. (2010). Immunological studies on amaranth sunset yellow and curcumin as food coloring agents in albino rats. Food and Chemical Toxicology, 48(6), 1581-1586. http://dx.doi.org/10.1016/j. fct.2010.03.028. PMid:20332010.

Hayes, A. W. (1994). Principles and Methods of Toxicology (3rd Ed., pp.687). Florida, USA: Raven Press Ltd.

Himri, I., Bellahcen, S., Souna, F., Belmekki, F., Aziz, M., \& Bnouham, M. (2011). A 90-day oral toxicity study of tartrazine, a synthetic food dye, in wistar rats. International Journal of Pharmacy and Pharmaceutical Sciences, 3, 159-169.

Jabeen, F., Rizvi, H. A., Aziz, F., \& Wasti, A. Z. (2013). Hyperglycemic induced variations in hematological indices in type 2 diabetic. International Journal of Advanced Research, 1, 322-334.

Jeannine, D. (2003). The impact of perceptual interactions on perceived flavor. Food Quality and Preference, 14(3), 137-146.

Jonnalagadda, P. R., Rao, P., Bhat, R. V., \& Nadamuni Naidu, A. (2004. (2004). Type andextent of colors used in Ready-to-eat (RTE) foods prepared in the non industrial sectors. International Journal of Food Science \& Technology, 39(2), 125-13. http://dx.doi.org/10.1046/j.09505423.2003.00749.x.

Khayyat, L., Essawy, A., Sorour, J., \& Soffar, A. (2017). Tartrazine induces structural and functional aberrations and genotoxic effect in vivo. PeerJ, 5, 3041. http://dx.doi.org/10.7717/peerj.3041. PMid:28243541.

Khatoon, A., Khan, M. Z., Zargham Khan, A., Saleemi, M. K., \& Javed, I. (2013). Amelioration of Ochratoxin A-induced immunotoxic effects by silymarin and vitamin $\mathrm{E}$ in White Leghorn cockerels. Journal of Immunotoxicology, 10(1), 25-31. http://dx.doi.org/10.3 109/1547691X.2012.686533 PMid:22734832.

Kumar, V. P., Madhu, C., Mannem, K., Asha, V. S., Rao, S., \& Prasad, M. S. (2012). Quantitative evaluation of carbohydrate levels in fruits by UV-visible Spectrophotometer. Asian. The Journal of Pharmacy Technology, 2(3), 99-100.

Lin, S., Chagnaadorj, A., Bayarsengee, U., Leung, T., \& Cheng, C. (2019). The compound, diallyl disulfide, enriched in garlic, prevents the progression of doxorubicin-induced nephropathy. Food Science and Technology, 39(4), 1040-1046. http://dx.doi.org/10.1590/fst.15418.

Mehedi, N., Ainad-Tabe, S., Mokrane, N., Addou, S., Zaoui, C., Kheroua, O., \& Saidi, D. (2009). Reproductive toxicology of tartrazine (FD and C Yellow No. 5) in Swiss albino mice. American Journal of Pharmacology and Toxicology, 4(4), 130-135. http://dx.doi.org/10.3844/ ajptsp.2009.130.135.
Milagres, M. P., Silva, D. M., Pereira, I. O., Senhorinho, L. M., Goulart Sant'Ana, A. E., \& Matos, T. B. (2020). Health benefits of chocolate consumption with high concentration of cocoa incorporated from triterpenic acids, isolated from Mansoa Hirsuta DC. Food Science and Technology, 40(Suppl. 1), 305-311. http://dx.doi.org/10.1590/fst.10519.

Montejo, J. F., Mondonedo, J. A. B., Lee, M. G. A., Ples, M. B., \& Vitor, R. J. S. 2nd (2015). Hematological effects of Ipomoea batatas (camote) and Phyllanthus niruri (sampa-sampalukan) from Philippines in the ICR mice (Mus musculus). Pacific Journal of Tropical Biomedicine, 5(1), 29-33. http://dx.doi.org/10.1016/S2221-1691(15)30166-0.

Nair, A. B., \& Jacob, S. (2016). A simple practice guide for dose conversion between animals and human. Journal of Basic and Clinical Pharmacy, 7(2), 27-31. http://dx.doi.org/10.4103/0976-0105.177703. PMid:27057123.

National Institute for Occupational Safety and Health - NIOSH. (2011). National Occupational Exposure Survey conducted from 1981-1983. Estimated numbers of employees potentially exposed to specific agents by 2-digit Standard Industrial Classification (SIC). Washington.

Oluwaniyi, O. O., Dosumu, O. O., Awolola, G. V., \& Abdulrahee, A. F. (2009). Nutritional analysis and stability studies of some satural and synthetic food colourants. American Journal of Food Technology, 4(5), 218-225. http://dx.doi.org/10.3923/ajft.2009.218.225.

Paliwal, P., Pancholi, S. S., \& Patel, R. K. (2011). Pharmacognostic parameters for evaluation of the rhizomes of Curcuma caesia. Journal of Advanced Pharmaceutical Technology \& Research, 2(1), 56-61. http://dx.doi.org/10.4103/2231-4040.79811. PMid:22171294.

Protima, R. (2008). Risk assessment of synthetic color: a case study in hydrabad. Journal of Nutrition \& Food Sciences, 4, 13.

Qiang, Z. (2015). Effects of extraction solvents on phytochemicals and antioxidant activities of walnut (juglans regia l.) green husk extracts. European Journal of Food Science and Technology, 3(5), 15-21.

Rajesh, H., Rao, S. N., Megha Rani, N., Prathima, K., Shetty Rajesh, E. P., \& Chandrashekhar, R. (2013). Phytochemical Analysis of Methanolic extract of Curcuma longa Linn. International Journal of Universal Pharmacy and Bio Sciences, 2(2), 39-45.

Rao, P., Bhat, R. V., Sudershan, R. V., \& Prasanna Krishna, T. (2006). Consumption of synthetic food colours during festivals in Hyderabad, India. British Food Journal, 107(5), 276-284. http:// dx.doi.org/10.1108/00070700510596875.

Ravindran, J., Prasad, S., \& Aggarwal, B. B. (2009). Curcumin and cancer cells: how many ways can curry kill tumor cells selectively? The AAPS Journal, 11(3), 495-510. http://dx.doi.org/10.1208/s12248009-9128-x. PMid:19590964.

Ronis, M. J. J., \& Cunny, H. C. (2001). Physiological (endogenous) factors affecting xenobiotic metabolism. In E. Hodgson \& R. C. Smart (Eds.), Introduction to biochemical toxicology (3rd ed. pp. 137-162). New York: Wiley Interscience.

Saivenkatesh, K., \& Murthy, J. S. R. (2016). Preliminary phytochemical profiles of different solvent extracts of leaves of Cassia hirsuta 1., a medicinal plant from Chittoor Dist., A.P. International Journal of Pharmacy and Pharmaceutical Sciences, 3(6), 18-23.

Sasaki, Y. F., Kawaguchi, S., Kamaya, A., Ohshita, M., Kabasawa, K., Iwama, K., Taniguchi, K., \& Tsuda, S. (2002). The comet assay with 8 mouse organs: results with 39 currently used food additives. Mutation Research, 519(1-2), 103-119. http://dx.doi.org/10.1016/ S1383-5718(02)00128-6. PMid:12160896.

Sathyaprabha, G., Kumaravel, S., \& Panneerselvam, A. (2011). Analysis of antioxidant activity, total phenol, total flavonoid and screening of phyto-components in Pleurotus platypus and Pleuro tuseous. Journal of Chemical and Pharmaceutical Research, 3(6), 1-6. 
Savithramma, N., Rao, M. L., \& Suhrulatha, D. (2011). Screening of medicinal plants for secondary metabolites. Middle East Journal of Scientific Research, 8(3), 579-584.

Saxena, J., \& Rajeshwari, S. (2012). Evaluation of phytochemical constituents in conventional and non-conventional species of Curcuma. International Research Journal of Pharmacy, 3(8), 203-204.

Shabi, R. R., Kumari, V., \& Chitarasu, T. (2014). Qualitative phytochemical characterization of thorn extracts of Canthium parviflorum Lam. International Research Journal of Pharmaceutical and Applied Sciences, 4(5), 13-17.

Shamsa, F. S., Monsef, H., Ghamooshi, R., \& Rizi, M. V. (2008). Spectrophotometric determination of total alkaloids in some Iranian medicinal plant. Thaiphesatchasan, 32, 17-20.

Sharma, R. A., Euden, S. A., Platton, S. L., Cooke, D. N., Shafayat, A., Hewitt, H. R., Marczylo, T. H., Morgan, B., Hemingway, D., Plummer, S. M., Pirmohamed, M., Gescher, A. J., \& Steward, W. P. (2004). Phase I clinical trial of oral curcum.in: biomarkers of systemic activity and compliance. Clinical Cancer Research, 10(20), 6847-6854. http:// dx.doi.org/10.1158/1078-0432.CCR-04-0744 PMid:15501961.

Sharma, A., Goyal, R. P., Chakravarty, G., \& Sharma, S. (2005). Haemotoxic effects of chocolate brown, a commonly used blend of permitted food colour on Swiss albino mice. Asian Journal of Experimental Sciences, 9, 93-103.

Sharma, G., Gautam, D., \& Goyal, R. P. (2009). Tartrazine induced hematological and serological changes in female Swiss albino mice, Musmusculus. Pharmacologyonline, 3, 774-788.

Soltan, S. S. A., \& Shehata, M. M. E. M. (2012). The effects of using color foods of children on immunity properties and liver, kidney on rats. Food and Nutrition Sciences, 3(7), 897-904. http://dx.doi. org/10.4236/fns.2012.37119.

Sharma, G. (2015). Reproductive toxic effects of the synthetic food dye kesari powder in female swiss albino mice (mus musculus). International Journal of Science. Technology and Management, 4, 153-168.

Stevens, L. J., Kuczek, T., Burgess, J. R., Stochelski, M. A., Arnold, L. E., \& Galland, L. (2013). Mechanisms of behavioral, atopic, and other reactions to artificial food colors in children. Nutrition Reviews, 71(5), 268-281. http://dx.doi.org/10.1111/nure.12023. PMid:23590704.

Stock, W., \& Hoffman, R. (2000). White blood cell 1: non-malignant disorders. Lancet, 355(9212), 1351-1357. http://dx.doi.org/10.1016/ S0140-6736(00)02125-5. PMid:10776761.

Swadhini, S. P., Santosh, R., Uma, C., Mythili, S., \& Sathiavelu, A. (2011). Phytochemical screening and antimicrobial activity of five medicinal plants against Myrothecium SP. International Journal of Pharma and Bio Sciences, 2(1), 272-279.

Tamilselvi, N., Krishnamoorthy, P., Dhamotharan, R., Arumugam, P., \& Sagadevan, E. (2012). Analysis of total phenols, total tannins and screening of phytocomponents in Indigo feraaspalathoides (Shivanar Vembu) Vah EX DC. Journal of Chemical and Pharmaceutical Research, 4(6), 3259-3262.

Tanaka, T. (2005). Reproductive and neurobehavioural toxicity study of trartrazine administration to mice in the diet. Food and Chemical Toxicology, 5, 16-25.

Timbrell, J. A. (2009). Principles of biochemical toxicology (4th ed.). USA: Informa Healthcare.

Tiwari, P., Kumar, B., Kaur, M., Kaur, G., \& Kaur, H. (2011). Phytochemical screening and extraction: a review. Internationale Pharmaceutica Sciencia, 1, 98-106.

U.S. Food and Drug Administration - FDA. (1985). Rule: Permanent listing of FD\&C Yellow No. 5. 50 Fed. Reg. 35774 (4 September 1985). St. Paul.

Veerachari, U., \& Bopaiah, A. K. (2011). Preliminary phytochemical evaluation of the leaf extract of five Cassia species. Journal of Chemical and Pharmaceutical Research, 3(5), 574-583.

Vijaykumar, M. H., Vaishampayan, P. A., Shouche, Y. S., \& Karegoudar, T. B. (2007). Decolourization of naphthalene-containing sulfonated azo dyes by Kerstesia sp. strain VKY1. Enzyme and Microbial Technology, 40(2), 204-211. http://dx.doi.org/10.1016/j. enzmictec.2006.04.001.

Vjibade, V. A., \& Egbebi, A. O. (2011). Effect of alkaloid extract of Phyllanthus niruri on rabbits infected with enteropathogenic escherichia coli. International Journal of Tropical Medicine and Public Health, 1(1), 33-39.

Vojdani, A., \& Vojdani, C. (2015). Immune reactivity to food coloring. Alternative Therapies in Health and Medicine, 21(Suppl. 1), 52-62. PMid:25599186.

Yoshitake, H., \& Sato, K. (1992). Effects of KW-2228 on the function of polymorphonuclear neutrophils in rats for the phagocytosis and killing activity and the production of active oxygen. The Japanese Journal of Antibiotics, 45(1), 112-120. PMid:1379650.

Zhang, L., Wang, F., \& Ren, X. (2018). Inhibitory effect of Dendrobium officinale polysaccharide on human gastric cancer cell xenografts in nude mice. Food Science and Technology, 38(1), 78-83. http://dx.doi. org/10.1590/1678-457x.00917. 